BBAMEM 75732

\title{
Labelling of liposomes with intercalating perylene fluorescent dyes
}

\author{
H. Schott ", D. Von Cunow a and H. Langhals " \\ "Institute of Organic Chemistry, Unicersity of Tübingen, Tübingen (Germany) \\ and "Institute of Organic Chemistry, University of Munich, Munich (Germany) \\ (Received 6 February 1992) \\ (Revised manuscript received 5 May 1992)
}

Key words: Perylene fluorescent dye; Fluorescent liposome; Anchor molecule; Ara C derivative; Immunoliposome

The high fluorescent potential and the exeeptional photostability of lipophilic derivatives of perylene-3.4:9,10-bis(dicarboximides) are utilized for the fluorescence-labelling of liposomes. The preparation of the liposomes is effected by supersonic starting from a lipid mixture consisting of the matrix lipids soy lecithin, cholesterol, $\alpha$-tocopher.s and the perylene dyes. From a multitude of perylene derivatives investigated only those are optimally incorporated into the bilayer membrane of unilamellar liposomes which are substituted at both nitrogen atoms by one or two linear hydrocarbon groups. In order to attain an optimal fluorescent quantum yield, about 200 to 300 dye molecules can be incorporated per liposome. The liposomes thus obtained have a diameter of about 70 to $80 \mathrm{~nm}$, are homogeneous and may be stored for more than seven months. Neither the fluorescent properties nor the stability of these liposomes are influenced by the additional incorporation of various ara C-derivatives and lipophilic anchor groups which subsequently enable the coupling of antibodies to the liposomes. As the water-insoluble perylene dyes are incorporated into the bilayer membrane, the aqueous inner volume of the liposomes remains available for a further utilization.

\section{Introduction}

The immunofluorescence technique is an approved method which has been developed particularly for use in biochemical analytics. As a standard procedure, antibodics each loaded with 2-5 molecules of a fluorescent dye are used for the detection of corresponding antigens. As the dye/antibody ratio is low, application of said fluorescent antibodies is not advisable in case the number of antigens to be detected is very small.

Truneh et al. $[1,2]$ have avoided this fundamental disadvantage of the prevailing immunofluorescence technique by coupling monoclonal antibodies to liposomes which have several hundred molecules of a fluorescent dye encapsulated in their aqueous inner volume. With the help of these fluorescent immunoliposomes even such antigens can be detected which cannot be visualized by fluorescent antibodies but only by an expensive fluorescence multiplication technique.

Correspondence to: $\mathrm{H}$. Schott, Institute of Organic Chemistry, University of Tübingen, Auf der Morgenstelle 18, D-7400 Tübingen, Germany.

Abbreviations: ara C, 1- $\beta$-D-arabinofuranosylcytosine; PBS, phosphate-buffered saline; SPC, soy phosphatıdylcholine.
Instead of encapsulating a water-soiubie dye in the aqueous inner volume we have incorporated the lipophilic perylene dye $N, N^{\prime}$-bis-(1-hexylheptyl)3,4:9,1-perylenebis(dicarboximide) into the bilayer membrane of unilamellar vesicles in order to obtain fluorescent liposomes. In the following, we shall report to what extent the respective structure of a perylene dye influences the incorporation of the dye into the liposomal membrane and the stability of the liposomes. Furthermore, it is investigated whether the incorporation of various lipophilic anchor molecules and ara C-derivatives affects the quality of the fluorescent liposomes.

\section{Materials and Methods}

\section{Materials}

The lipid soy phosphatidylcholine (SPC, Epikuron 200) is obtained from L. Meyer, Hamburg, Germany, cholesterol is from Fluka, Buchs, Switzerland; DI.- $\alpha$ tocopherol and the organic solvents are from Merck, Darmstadt, Germany.

The perylene dyes are prepared from perylene3,4:9,10-tetracarboxylic bisanhydride, the corresponding amine or amine hydrochloride and imidazole ac- 
cording to literature procedure [4-9]. Special care is taken to prepare highly pure dyes.

The anchor molecules are synthesized as described; $N$-palmitoylcysteamine [11], $N^{2}$-palmitoyl-L-lysine methyl ester $\cdot \mathrm{HCl}$ [12], $N^{2}$-palmitoyl- $N^{6}$-succinoyl-Llysine [12]. $N^{2}$-Palmitoyl- $N^{6}$-(3-maleinimidopropionyl)$\mathrm{L}$-lysine methyl ester is prepared in analogy to the published synthesis of $N^{2}$-palmitoyl- $N^{6}$-(6-maleinimidocaproyl)-L-lysine methyl ester [3] and $N$-biotinylphosphatidylethanolamide according to Ref. 13.

\section{Methods}

1. Preparation of fluorescent liposomes. A mixture consisting of $100 \mathrm{mg}$ SPC, $10 \mathrm{mg}$ cholesterol and $1 \mathrm{mg}$ D,l-a - tocopherol is filled up with chloroform/methanol (1: $1, v / v)$ to a volume of $1 \mathrm{ml}$. To $600 \mu \mathrm{l}$ of this lipid solution, $100-600 \mu \mathrm{l}$ of a solution containing one of the various fluorescent dyes listed in Fig. 1 are added. Said dye solution is prepared by adding $200 \mu \mathrm{l}$ of chloroform to up to $1 \mathrm{mg}$ of the respective dye and subsequent sonification at room temperature in a supersonic bath (Elma, Singen, Germany). The dyes differ in solubility and in case of a perylene derivative which is not completely solubilized under the conditions described above, a further $200 \mu \mathrm{l}$ of chloroform are added and sonified again. By this method dye solutions with or without precipitate are obtained, 100 $\mu l$ of which are added to $600 \mu l$ of the lipid solution. The solvent of the combined solutions is blown off with air in a $10 \mathrm{ml}$ test tube at $50^{\circ} \mathrm{C}$ and the lipid film that remains behind is evaporated to dryness for $1 \mathrm{th}$ at $50^{\circ} \mathrm{C}$ at a water jet aspirator. Then the lipid film is suspended in $3 \mathrm{ml}$ of $10 \mathrm{mM}$ PBS $(0.9 \% \mathrm{NaCl}+10 \mathrm{mM}$ $\mathrm{NaH}_{2} \mathrm{PO}_{4}$ ), $\mathrm{pH} 7.4$ and sonified (40) W) for $30 \mathrm{~min}$ with a micro tip at a Branson Sonifier B-12 (Branson-Sonic Power, USA). The cooled lipusome dispersion is filtered through a $0.2 \mu \mathrm{m}$ sterile filter, dialyzed thoroughly against PBS and stored at $4^{\circ} \mathrm{C}$ until further use.

2. Proparation of fluorescent liposomes dericatized with anchor molecules. A mixture consisting of the matrix lipids $100 \mathrm{mg}$ SPC, $10 \mathrm{mg}$ cholesterol, $1 \mathrm{mg}$ $D, 1,-\alpha$-tocopherol and one of the five anchor molecules shown in Fig. 3 (1 mg $N$-palmitoylcysteamine (1), $4 \mathrm{mg}$ $N^{2}$-palmitoyl-t.-lysine methyl ester $\cdot \mathrm{HCl}[2], 7 \mathrm{mg} N^{2}$ palmitoyl- $N^{6}$-succinoyl-L-lysine (3), $2 \mathrm{mg} N^{2}$-palmitoyl$N^{6}$ (3-mateinimidopropionyl)-L. lysine methyl ester (4), $0.1 \mathrm{mg} N$-biotinylphosphatidylethanolamide (5)) is filled up with chloroform/methanol $(1: 1, v / v)$ to a volume of $1 \mathrm{ml}$, resulting in the lipid stock solution. 1-2.5 $\mathrm{mg}$ of the dyes $S-13$ or $S-17$ are dissolved in $1 \mathrm{ml}$ of chloroform and 150-600 $\mu$ l thereof are added to the $600 \mu \mathrm{l}$ of the lipid stock solution. As described above, the solvent of the combined solutions is blown off with air (in the presence of $N$-palmitoylcysteamine (1) or $N^{2}$-palmitoyl- $N^{6}$-(3-maleinimidopropionyl)-L-lysine methyl ester (4) $N_{2}$ is used and the procedure is performed in the dark) and the remaining lipid film is transformed into fluorescent liposomes derivatized with anchor molecules.

3. Preparation of fluorescent liposomes derivatized with anchor molecules and loaded with ara $C$-dericatives. A mixture consisting of the matrix lipids $100 \mathrm{mg}$ SPC, $10 \mathrm{mg}$ cholesterol, $1 \mathrm{mg}$ D, L- $\alpha$-tocopherol and 7 mg $N^{2}$-palmitoyl- $N^{6}$-succinoyl-L.-lysine (3) is filled up with chloroform/methanol $(1: 1, v / v)$ to a volume of 1 $\mathrm{ml}$ resulting in the lipid stock solution. 1-2.5 $\mathrm{mg}$ of the dyes S-13 or S-17 are dissolved in $1 \mathrm{ml}$ of chloroform and 150-600 $\mu$ l thereof are added to $600 \mu \mu$ l of the lipid stock solution. $7.5 \mathrm{mg}$ of a water-soluble or lipophilic 1- $\beta$-1)-arabinofuranosylcytosine derivative $[15,16]$ or mixtures of different ara C-derivatives dissolved or suspended in chloroform are added. The solvent of the combined solutions is blown off and the resulting lipid film is transformed into the liposome dispersion as described above.

For the purpose of determining the diameter and the polydispersity the liposome dispersion is diluted with PBS to a concentration of about $2 \mathrm{mg} \mathrm{SPC} / \mathrm{ml}$ and measured in a Coulter Nano-Sizer (Coulter Electronics, Harpenden Hearts, UK). Both parameters are measured regularly about every ten days.

For the recording of the fluorescence spectra depicted in Fig. 2 the liposome dispersions are diluted with degassed PBS according to the data given in the legend and measured with a spectrofluorometer JY3 (Jobin Yvon, Long Jumcau, France, in combination with calculator Hewlett Packard 9815 A and plotter Hewlett Packard 7.225) at $489 \mathrm{~nm}$ excitation at $25^{\circ} \mathrm{C}$, concentration factor 1 .

\section{Results}

The high fluorescent potential and exceptional photostability of perylene-3,4:9,10-bis(dicarboximides) in solution have been investigated after the introduction of the solubility increasing dialkylphenylmethyl phenyl groups or long-chain secondary alkyl groups at the nitrogen atoms of the aromatic system [6-9]. The substituents at the nitrogen atoms of the perylene disivatives (see Fig. 1) can be varied without losing the good optical properties of the dyes. In orientating studies it is first determined which of the perylene derivatives listed in Fig. 1 are incorporated into the membrane of inilamellar liposomes and in what way the incorporation affects the stability of the liposomes. For this purpose, a mixture of so-called matrix components (SPC/cholesterol/D,L- $\alpha$-tocopherol) in a weight ratio of 10:1:0.1 is dissolved in the smallest possible volume of chloroform/methanol (1: 1, v/v). By the addition of the antioxidant $\alpha$-tocopherol the unsaturated carboxylic acid residues of the lipids are protected from oxidation. By this means the liposomes become more 
stable and remain in a better condition during storage over an extended period of time. To this solution, the perylene derivative dissolved in chloroform is added. In the case of perylene derivatives which are hardly solu-
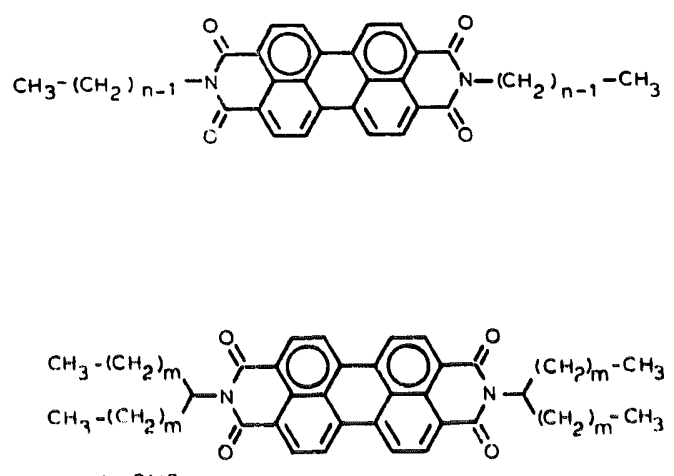

$m=(n-3) / 2$
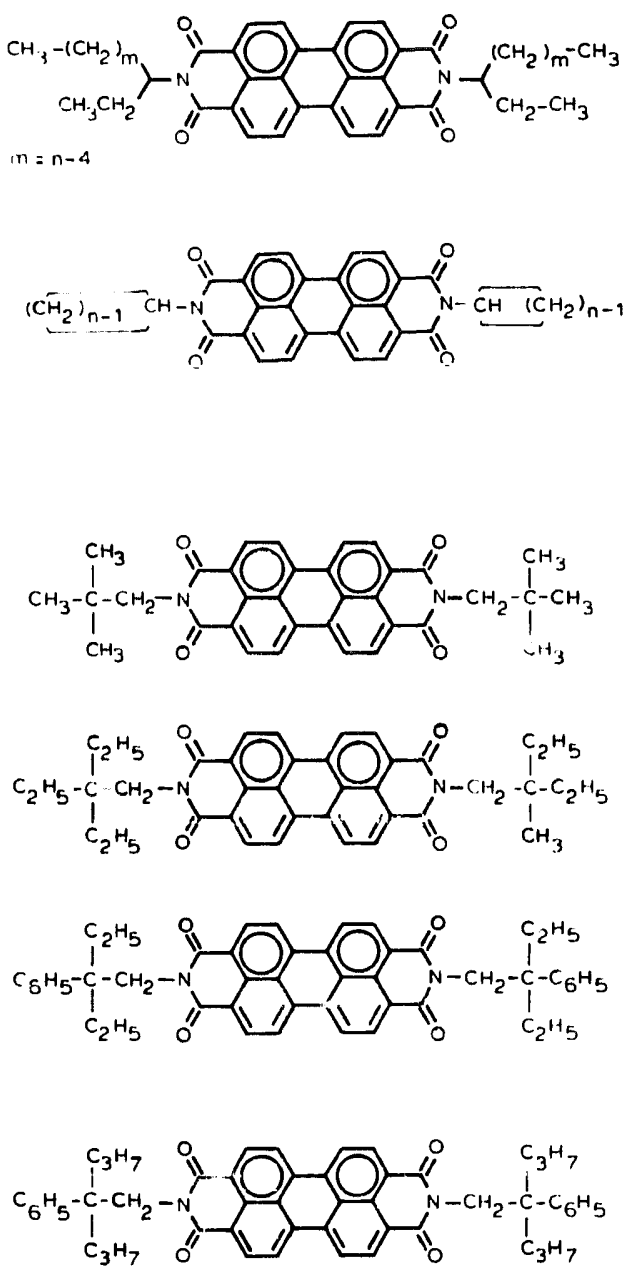

ble, the supernatant from a saturated chloroform mixture is used.

For subsequent liposome formation it is crucial that the matrix lipids being the chief constituents of the

\begin{tabular}{c|cc}
$N-n$ & M.W. & $\varepsilon$ \\
\hline$N-3$ & $\mathbf{4 7 4 . 5}$ & $\mathbf{7 8 7 3 0}$ \\
$N-4$ & 502.6 & 83050 \\
$N-5$ & 530.6 & 84020 \\
$N-6$ & $\mathbf{5 5 8 . 7}$ & 85700 \\
$N-9$ & 642.8 & 85370 \\
$N-12$ & 727.0 & 85150 \\
$N-14$ & 783.1 & 85120 \\
$N-16$ & 839.2 & 82470 \\
$N-18$ & 895.3 & 86100
\end{tabular}

\begin{tabular}{l|rc} 
S-n & M.W. & $E$ \\
\hline $5-3$ & 475.5 & 83000 \\
S-5 & 530.6 & 83560 \\
S-7 & 586.7 & 85510 \\
S-9 & 642.8 & 85510 \\
S-11 & 698.9 & 89950 \\
S-13 & 755.1 & 91200 \\
S-15 & 811.2 & 86040 \\
S-17 & 867.3 & 85650 \\
S-19 & 922.7 & 87080 \\
S-21 & 978.8 & 84800 \\
S-25 & 1090.9 & 84450 \\
S-37 & 1428.3 & 84400
\end{tabular}

\begin{tabular}{l|lc} 
AE-n & M.W. & $\varepsilon$ \\
\hline$A E-7$ & 586.7 & 83860 \\
$A E-9$ & 642.8 & 82290 \\
$A E-11$ & 698.9 & 89580
\end{tabular}

\begin{tabular}{l|cc} 
C-n & M.W. & $\varepsilon$ \\
\hline C-3 & 470.5 & 79420 \\
C-4 & 498.5 & 81010 \\
C-5 & 526.6 & 83190 \\
C-6 & 554.6 & 85000 \\
C-7 & 582.7 & 84220 \\
C-8 & 610.8 & 85060 \\
C-9 & 638.8 & 85840 \\
C-10 & 656.9 & 85810 \\
C-11 & 694.9 & 84570 \\
C-12 & 723.0 & 85460 \\
C-13 & 751.0 & 85310 \\
C-14 & 779.1 & 84920 \\
C-15 & 807.1 & 85900 \\
C-16 & 835.2 & 86230
\end{tabular}

TCH $1,1.1$

M.W. . 530.6

M.W. 2540

TCH 2.2.2

M.W. 614.8

E. 80960

PTCH 2.2

M.W. $=710.9$
$\varepsilon=77240$

PTCH 3,3

M.W. -767.0

ค. 79160

Fig. 1. Structural formulas and abbreviations of fluorescent perylene derivatives incorporated into liposomes. 
mixture are well soluble in the used solvent and that a homogeneous lipid film is obtained on concentration of the solution. Accordingly, all those solvents are unsuitable in which only the perylene dye is well, but the matrix lipids are poorly soluble.

After evaporation of the solution thus obtained to a lipid film, PBS is added in such an amount that $1 \mathrm{ml}$ PBS is available for $20 \mathrm{mg} \mathrm{SPC}, 2 \mathrm{mg}$ cholesterol, 0.2 $\mathrm{mg} \alpha$-tocopherol and up to $0.5 \mathrm{mg}$ perylene derivative, and a suspension is prepared therefrom by means of a supersonic bath. Subsequently, the suspension is sonified with a desintegrator until it turns into an opalescent liposome dispersion. In doing so, it becomes evident that all perylene derivatives listed in Fig. I can be incorporated into liposomes quite well but the stability of the liposomes obtained is largely dependent on the respective dye incorporated, most of the dyes having a destabilizing effect.

Due to the incorporation of perylene derivatives which are substituted at both nitrogen atoms with cyclic $\mathrm{C}_{3}-\mathrm{C}_{10^{-}}$and considerably branched (PTCH-, TCH-derivatives) hydrocarbon groups, or hydrocarbon residues which are either too short or too long $(N$ 3,5,6,9,12,16,18-; AE-7,9-; S-3,5,7,9- derivatives), the bilayer membranes are destabilized to such an extent that in some liposome preparations turbidities, flocculations and precipiates already occur within several hours or days and many preparations desintegrate within one month. Accordingly, these perylene derivatives are unsuitable for the fluorescence labelling of unilamellar liposomes. On the other side, perylene derivatives carrying at both nitrogen atoms one or two linear hydrocarbon groups which well fit into the lipid matrix, do either not at all (S-11,13,15,17,21,25,37-derivatives) or only to a small extent ( $N-4,14-;$ AE-11-derivatives) influence the stability of the liposomes.

By means of a Nano-Sizer the polydispersity $(H)$ and the diameter $(D)$ are automatically determined for all of the liposome dispersions. The polydispersity is given on a scale ranging from 0 to 9 , with 0 indicating a very homogeneous and 9 a very inhomogeneous dispersion. The first measurement is carried out immediately after the preparation of the liposomes. During storage of the liposome dispersions at $4^{\circ} \mathrm{C}$ the polydispersity and diameter are checked regularly at intervals of about ten days. As long as the values do not change significantly the liposomes are regarded as being stable. Visible flocculation in the liposome dispersions during storage indicates that the liposomes become unstable. Usually, the formation of flocks is associated

\section{TABLE I}

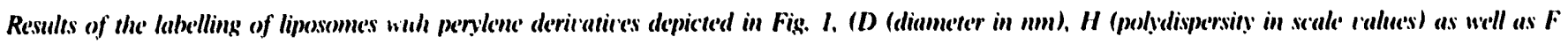
(flecculation) aficer no days diuring storage at $f^{\circ} \mathrm{C}$

\begin{tabular}{|c|c|c|c|c|c|c|c|}
\hline \multirow{2}{*}{\multicolumn{2}{|c|}{$\begin{array}{l}\text { Perylene derivative } \\
\text { employed per ml PBS }\end{array}$}} & \multirow{3}{*}{$\begin{array}{l}\text { Lipid mixture } \\
\text { ner ml PBS } \\
\text { No, " }\end{array}$} & \multicolumn{2}{|l|}{ Liposome preparation } & \multicolumn{3}{|c|}{ Ohserved } \\
\hline & & & \multirow{2}{*}{$\begin{array}{l}\text { calculated } \\
\text { liposomes }\left(x \cdot 10^{1+}\right) \\
\text { per ml PBS }\end{array}$} & \multirow{2}{*}{$\begin{array}{l}\text { dye molecules } \\
\text { per liposone }\end{array}$} & \multirow[t]{2}{*}{$\bar{D}$} & \multirow[t]{2}{*}{$H$} & \multirow[t]{2}{*}{ I } \\
\hline name & $\begin{array}{l}\text { amount } \\
(\mu g)\end{array}$ & & & & & & \\
\hline$A E=11$ & 50 & 1 & 1.4 & 310 & 124 & 3 & 31) \\
\hline $\mathrm{N}=4$ & 3 & 1 & 1.4 & 30 & 122 & 3 & 30 \\
\hline N.14 & 2 & 1 & 1.4 & 10 & 123 & 2 & 30 \\
\hline$S-11$ & 50 & i & 2.0 & 220 & 90 & 4 & 100 \\
\hline$S=13$ & 50 & 1 & 2.3 & 170 & 102 & 2 & 120 \\
\hline$S=13$ & 51) & 2 & 5.0 & 80 & 72 & 3 & 120 \\
\hline$S=15$ & 100 & 1 & 1.4 & 530 & 125 & 2 & 80 \\
\hline$S=17$ & 50 & 1 & 1.5 & 230 & 110 & 2 & 220 \\
\hline \multirow[t]{4}{*}{ S.17 } & 50 & 2 & 5.0 & 70 & 72 & 1 & $>220$ \\
\hline & $1(x)$ & 2 & 3.8 & 180 & 76 & 2 & $>220$ \\
\hline & $2(x)$ & 2 & 5.0 & 280 & 70 & 2 & $>220$ \\
\hline & $s(0)$ & 2 & 3.9 & 900 & 79 & 3 & $>220$ \\
\hline 5.21 & 41 & 1 & 2.0 & 120 & 100 & 2 & 220 \\
\hline S.25 & 30 & 1 & 2.0 & 80 & 100 & 2 & 220 \\
\hline S.27 & 20 & 1 & 2.0 & 40 & 106 & 2 & 110 \\
\hline
\end{tabular}

" No. indicates the employed amount of total lipid exclusive of the amount of perylene derivative: $1=22.2 \mathrm{mg} \mathrm{(20)} \mathrm{mg} \mathrm{SPC.} 2 \mathrm{mg}$ cholesterol, 0.2 mg $\alpha$-1(ccopherol); $2=26.1 \mathrm{mg}\left(20 \mathrm{mg} \mathrm{SPC}, 2 \mathrm{mg}\right.$ cholesterol, $0.2 \mathrm{mg} \alpha$-tocopherol, $1.4 \mathrm{mg} N^{2}$-palmitoyl- $N^{6}$-succinoyl-1.-lysine, $2.5 \mathrm{mg}$ ara C-derivative.

n The number of liposomes formed is calculated from the liposomal diameters and from the assumptions on liposome geometry parameters as made by Huang and Mason [10]. For example, if spherical liposomes having a diameter of $100 \mathrm{~nm}$ are prepared starting from $1 \mathrm{mg}$ SPC, according to Huang and Mason $1 \mathrm{ml}$ of the obtained dispersion contains approx. $9.11 \cdot 10^{12}$ liposomes. Since in our preparations total lipid (SPC, cholesterol, $\alpha$-tocopherol, fluorescent dye, and optionally, anchor molecules and ara C-derivative) is up to $26.6 \mathrm{mg}$ per ml dispersion, e.g.. about $2.4 \cdot 10^{14}(D=100 \mathrm{~nm})$ are contained therein. The values given are built on the assumption that all membrane constituting molecules equal SPC in volume $\left(1.253 \mathrm{~nm}^{3}\right)$. 
with a marked increase of vesicle size and polydispersity. On the first appearance of flocks the measurements are stopped and the liposomes are discarded.

In Table I, only those values are presented which have been measured for liposome dispersions showing no changes during storage for at least $\mathbf{3 0}$ days. For the liposome dispersions specified in Table I the polydispersity determined ranges from 1 to 4 , whereas the diameter of the liposomes ranges from about 70 to about $125 \mathrm{~nm}$, thus corresponding to the size of unilamellar liposomes. As the perylene dyes S-13 and S-17 yield the best rates of incorporation combined with a high stability of the obtained liposomes the following extended investigations are restricted to these dyes.

In order to optimize the concentration of the fluorochrome to be incorporated into the liposomal bilayer the fluorescence signal in dependence of increasing concentrations is measured in a spectrofluorometer for the perylene derivatives $S-13$ and S-17, respectively. From Fig. 2, it is evident that the increase in fluorescence signal is not directly proportional to the concentration of the perylene derivative incorporated. In the range from 50 to $100 \mu \mathrm{g}$ of dye per $\mathrm{ml}$ liposome dispersion the increase of the intensity of emission is approximately linear with increasing dye concentrations (see spectra 1, 2 and 4). On comparison of spectrum 4 with spectrum 6 , it is evident that at equal amounts of incorporated dye and at the same dilution, the fluorescence signal produced by S-17 is slightly higher than that of S-13. The incorporation of amounts of dye exceeding $100 \mu \mathrm{g}$ is inexpedient because it is not correlated with a correspondingly high fluorescence signal but leads to a quench effect. Said quench effect cannot be neutralized by dilution of the liposome dispersion as is clearly evidenced by the comparison of spectrum 1 with spectrum 4 . Furthermore, this finding shows that the liposomes contain stably incorporated dye which cannot be released into the medium by dilution of the liposome dispersion. The concentration of fluorochromes in the liposomal membrane can be easily manipulated to yield the desired intensity of the fluorescence signal.

Fluorescent liposomes having functional groups for the immobilization of antibodies have been prepared by incorporating into liposomes various lipophilic anchor molecules together with the perylene dyes $S-1.3$ or S-17. A selection of anchor molecules which have been used for the functionalization of liposomes in our previous studies $[3,11,12,14]$ is specified in Fig. 3. For the preparation of fluorescent liposomes derivatized with functional groups the respective anchor molecules are added to the lipid mixture consisting of the matrix lipids and the respective dye in an amount of 0.02 to $1.4 \mathrm{mg}$ per $20 \mathrm{mg} \mathrm{SPC}$ and the lipid mixture is transformed into a liposome dispersion. Dependent on the nature and amount of anchor molecules incorporated the bilayer membrane of the liposomes thus obtained contains a definite number of mercapto-, amino-, carboxylic, maleinimido- or biotinyl residues. Diameter, polydispersity and stability of these liposomes are found to be essentially determined by the nature and amount of the incorporated anchor molecule, as the respective values obtained for the fluorescent, derivatized liposomes do not differ from those obtained for the corre-

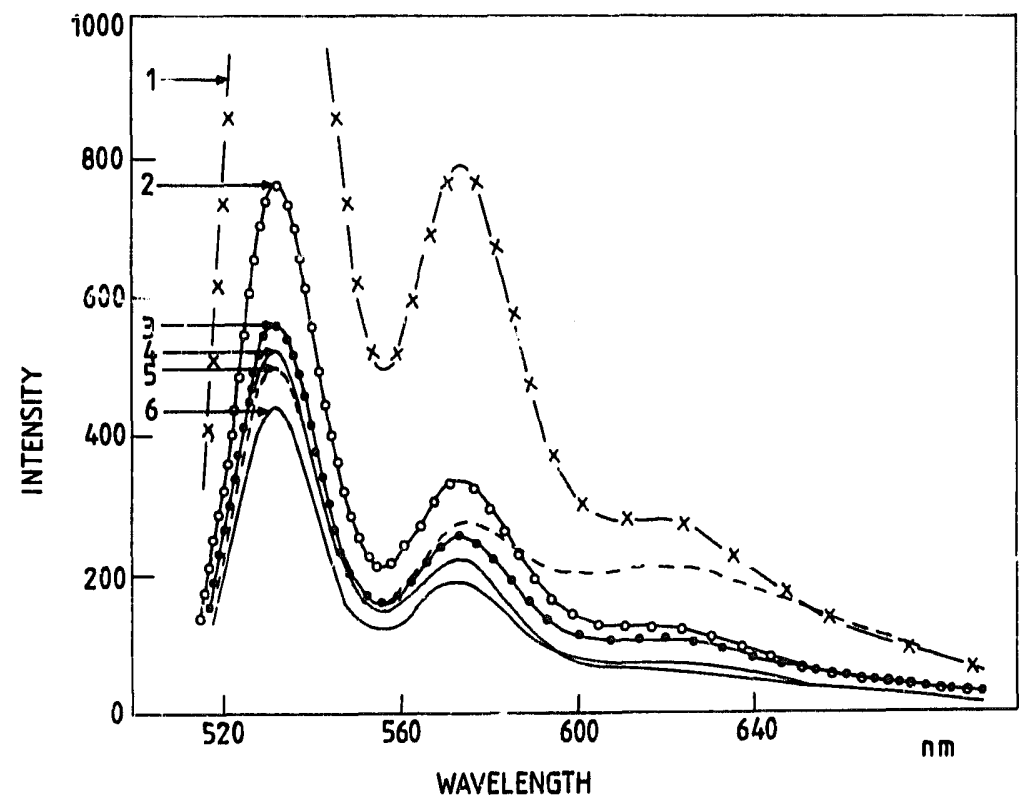

Fig. 2. Emission spectra of fluorescent liposomes ( $D: 70-79 \mathrm{~nm}, H: 1-3$ ) loaded with different amounts of perylene derivatives $S-17$ (spectra $1-5$ ) and S-13 (spectrum 6) after excitation at $489 \mathrm{~nm}$. Spectrum 1 is obtained for $1 \mathrm{ml}$ of a dispersion, having incorporated into the liposomes 50) $\mu \mathrm{g}$ of S-17 and being measured at a dilution of $1: 100$. Spectra 2-6 are obtained of the following dispersions and dilutions, respectively: 2 , 100$) !: 8$ $\mathrm{S}-17 / 1: 400 ; 3,200 \mu \mathrm{g} \mathrm{S}-17 / 1: 400 ; 4,50 \mu \mathrm{g} \mathrm{S}-17 / 1: 400 ; 5,500 \mu \mathrm{g} \mathrm{S}-17 / 1: 400 ; 6,50 \mu \mathrm{g} \mathrm{S}-13 / 1: 400$. 
(1)<smiles>O=C(S)NCCCS</smiles>

(2)

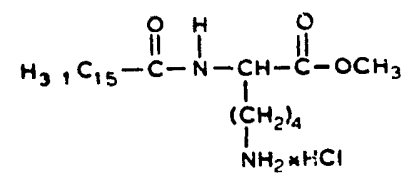

(3)

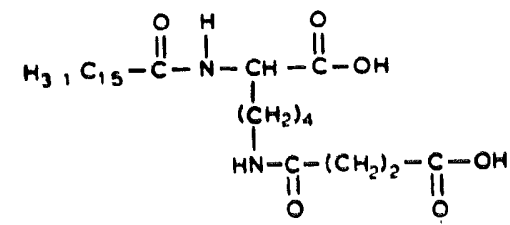

(4)<smiles>COC(=O)C(CCNC(=O)CN1C(=O)C=CC1=O)NC(=O)O</smiles>

(5)

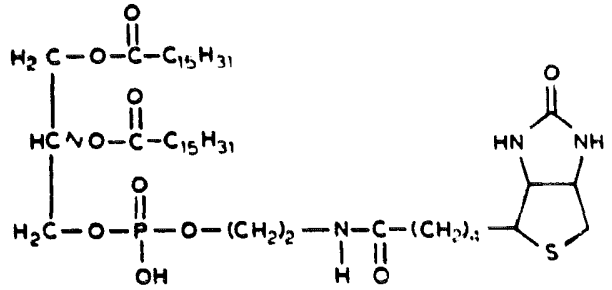

Fig. 3. Structural formulas of lipophilic anchor molecules incorporated into the bilayer membrane of fluorescent liposomes enabling a liposone:-antitody soupling. The antihodies ean he linked to the anchor molecules of the liposomes by disulfide- (1), amide-(2,3) and thiocther. (4) houds or hy avidin-biatin complexation.

sponding non-fluorescent liposomes. Compared to fluorescent non-derivatized liposomes the additional incorporation of an anchor molecule may result in a decrease of the liposomal diameter.

The preparation of fluorescent liposomes derivatized with anchor molecules and loaded with ara C-derivatives starts trom a mixture of matrix lipids to which the cytostatic drug is given in addition to the perylene derivative and the anchor molecule, and is also achieved by supersonic. Representative of this group of liposomes the data of liposome dispersions loaded with the anchor molecule (3) (cf. Fig. 3), a water-soluble and/or lipophilic derivative of the known cytostatic 1- $\beta$-1)arabinofuranosylcytosine and labelled with $S-13$ or $S-17$ are listed in Table 1 . It is remarkable that the incorporation of the anchor molecule (3) significantly increases the stability of the fluorescent liposumes. Liposomes containing only a fluorescent dye and ara C-derivatives flocculate earlier. The liposome dispersions exhibit the same properties as the corresponding non-labelled li- posomes we have disclosed in previous publications $[11,12,14]$. This finding aiso proves to be truc after the coupling of monoclonal antibodies to the functionalized fluorescent liposomes, which has been performed analogously to the way we have described previously [3].

All investigations show that the perylene derivatives S-13 and S-17 render possible an optimum fluorescence labelling of liposomes without deteriorating the properties of the liposome dispersions.

\section{Discussion}

In the present study it is demonstrated that certain perylene derivatives can be intercalated into the bilayer membrane of liposomes. According to this method which is easily practicable, very stable fluorescent liposomes are obtained taking advantage of the excellent photostability of the perylene derivatives. For the intercalation of the perylene derivatives it is decisive to what extent their conformation fits into the conformation of the matrix lipid. Unfavourable conformations do not only impede the incorporation but also lead to a destabilization of the entire lipid matrix. Of a perylene derivative with an unsuitable conformation a share of $0.5 \%$ of the total lipid mixture can result in complete destabilisation. On the contrary, the intercalation of perylene derivatives with a suitable conformation can easily be accomplished in sufficiently high amounts. Said substituents include dyes with swallow-tailed substituents and substituents with two long hydrocarbon chains as present in the derivatives $\mathrm{S}-13$ and $\mathrm{S}-17$ which exhibit optimal properties for the incorporation into liposomes concerning solubility and stability. For example, the incorporation of about 70 to about 9(0) molecules of S-17 per liposome results in liposomes with diameters ranging from 70 to $80 \mathrm{~nm}$, which remain stable without change for more than seven months.

The intercalation of the dyes into the lipid bilayer membrane is induced by their highly hydrophobic properties. The dyes which are insoluble in water produce orange fluorescent inedium in the presence of liposomes. A further indication for the intercalation is the fluorescence wavelength of these media, because the dyes are weakly solvatochromic [17]. The fluorescence maximum of $535 \mathrm{~nm}$ corresponds well with the maximum in chloroform (534 nm [18]), which is expected to be a medium with similar solvent effects compared to the lipid bilayer of the liposome solution (the fluorescent maximum of the dye is hypsochromically shifted to $523.5 \mathrm{~nm}$ in $\mathrm{n}$-heptane as a solvent with low polarity). Even the line form of the emission of the liposomes is the same as for the emission of the dyes in chloroform solution, whereas one observes an aggregation of the dyes [19] with a characteristic broadening of the spectrum in highly polar media like alcohols. The 
fluorescence emission of the liposomes is found to be independent of $\mathrm{pH}$. All these results are experimental evidences for the intercalation of the dyes.

The fluorescent liposomes can be loaded with drugs for therapeutical purposes. Water-soluble drugs, e.g., ara C-derivatives as used in our case, can be encapsulated in the aqueous inner volume whereas water-insoluble drugs, in our case lipophilic ara C-derivatives, are incorporated into the lipid membrane. The finding that the polydispersity as well as the diameter of these liposomes remain constant during storage evidences that the dye and the drugs are stably incorporated. Furthermore, stable incorporation of the drugs is indicated by spectroscopic analysis after repeated dialysis of the liposome dispersions. In the diffusate neither fluorescent dye nor ara C-derivatives can be spectroscopically detected and the adsorption of the liposome dispersion remains constant before and after dialysis. The fluoreseent liposomes loaded with ara C-derivatives can also be derivatized with different kinds of anchor molecules suitable for the immobilisation of antibodies [3,11,14,20-24].

For the binding of the immunoliposomes to a target cell usually one antibody coupled per liposome is sufficient. In comparison to traditional immunoconjugates carrying only 2 to 5 fluorescent molecules per antibody the fluorescence is enhanced by a factor of 100 which is a great advantage in practice. For analytical purposes these liposomes may be used as multicolour immunofluorescent reagents as in addition to the perylene derivatives intercalated in the bilayer membrane water-soluble fluorescent dyes may be encapsulated in the aqueous inner volume.

With our liposomes the frequently observed problem that hydrophobic dyes equilibrate with every hydrophobic environment available has not occurred for any of the examples investigated by us. The inert behaviour of the liposome dispersions can be demonstrated, e.g., by chromatography. The dispersion can be chromatographed on hydrophobic materials such as Sephadex and Ultrogel without noticeable losses. In contrast thereto, liposomes labelled with other lipophilic fluorescent dycs are partially or completely absorbed during chromatography. In case considerable adsorption occurs during chromatography, corresponding unspecific interaction of the liposomes is to be expected in cellular systems. Judging from these and previously published results [3], the incorporated perylene derivatives will not affect the cell-binding charac- teristics of the immunoliposomes. At most, at the target cell such an exchange of dye may happen when the liposomes are taken up by the target cells. This effect, however, is desired as it favors the labelling of the target cell.

As the perylene derivatives S-13 and S-17 have been shown to intercalate into the lipid bilayer membrane, our liposomes may also be useful for the monitoring of lipid mixing if membrane-fusion events actually take place.

\section{References}

1 Trunch, A., Machy, P. and Horan, P.K. (1987) J. Immunol. Methods 100, 59-71.

2 Trunch, A. and Machy, P. (1987) Cytometry 8, 562 .567.

3 Schwendener, R.A., Trüb, T., Schott, H., Langhals, H., Barth, R.F., Groscurtl, P. and Hengartner H. (190(0) Biochim. Biophys. Acta 1026, 69-79.

4 Langhals, H. (1985) Chem. Ber. 118, 4674-4681.

5 Demmig. S. and Langhals, H. (1988) (hem. Ber. 121, 225-2.3i)

6 Langhals, H., Demmig S. and Huher, H. (I98K) Spectrochim. Acta 44A, $1180-1193$.

7 Langhals, H. and Demmig, S. D.O.S. 40(17618.0 (March 10, 1990)).

8 Langhals, H. D.O.S. 4(137735.0 (November 27, 199()).

9 Löhmannsröben, H.-G. and Langhals, H. (1989) Appl. Phys. B 48. 449-452.

10 Huang, C. and Mason, J.T. (1978) Proc. Natl. Acad. Sci. USA 75. 308-310.

11 Schott, H., Seeling, R., Hengartner, H. and Schwendener, R.A. (1988) Biochim. Biophys. Acta 940, 127-135.

12 Schott, H., Heß, W., Hengartner, H. and Schwendener, R.A. (i988) Biochim. Biophys. Acta 943, 53-62.

13 Bayer, E.A., Rivnay, B. and Skutelsky, E. (1979) Biochim. Biophys. Acta 55(), 464-473.

14 Schott, H., Leitner, B., Schwendener, R.A. and Hengartner, H. (1988) J. Chromatogr. 441, 115-124.

15 The synthesis and structure of the new water-soluble ara C-derivatives will be published elsewlere.

16 Schwendener, R.A. and Schott, H. (1992) Int. J. Cancer, in press.

17 Langhals, H., (1991) in Similarity Models in Organic Chemistry. Biochemistry and Related Fields (Zalewski, R.I., Krygowski, T.M. and Shorter, J., eds.) pp. 283-342, Elsevier, Amsterdam.

18 Langhals, H., Demmig, S. and Porawa, T., (1991) J. Prakt. Chem. $333,733-748$.

19 Johansson, L.B.-A. and Langhals, H., (1991) Spectrochim. Acta 47A, 857-861.

20) Barbet, J., Machy, P. and Leserman, L.D. (1961) J. Supramul. Struct. Cell. Biochem. 16, 243-258.

21 Heath, T.D. (1987) Methods Enzymol. 149, 111-119.

22 Martin, F.J. and Papahadjopoulos, D. (1982) J. Biol. Chem. 257. 286-288.

23 Weckenmann, H.P., Matzku, S and Stricker, H. (1988) Arzneim.-Forsch./Drug Res. 38, 1156-1562.

24 Loughrey, H., Bally, M.B. and Cullis, P.R. (1987) Biochim. Biophys. Acta 901, 157-160. 